\title{
Flow diversion treatment of complex bifurcation aneurysms beyond the circle of Willis: complications, aneurysm sac occlusion, reabsorption, recurrence, and jailed branch modification at follow-up
}

\author{
Caterina Michelozzi, MD, ${ }^{1}$ Jean Darcourt, MD, ${ }^{1}$ Adrien Guenego, MD, ${ }^{1}$ \\ Anne-Christine Januel, MD, ${ }^{1}$ Philippe Tall, MD, ${ }^{1}$ Matthias Gawlitza, MD, ${ }^{2}$ \\ Fabrice Bonneville, MD, PhD, ${ }^{1}$ and Christophe Cognard, MD, $\mathrm{PhD}^{1}$ \\ 1Service de Neuroradiologie, Hôpital Pierre-Paul Riquet, CHU de Toulouse; and ${ }^{2}$ Service de Neuroradiologie, CHU Reims, \\ France
}

\begin{abstract}
OBJECTIVE The purpose of this study is to present the authors' medium-term results, with special emphasis on complications, occlusion rate of the aneurysm sac (digital subtraction angiography [DSA] and MRI), and the fate of cortical branches and perforating arteries covered ("jailed") by the flow diverter (FD) stent.

METHODS Between January 2010 and September 2017, 29 patients (14 female) with 30 aneurysms were treated with an FD stent. Twenty-one aneurysms were at the middle cerebral artery bifurcation, 8 were in the anterior communicating artery region, and 1 was a pericallosal artery bifurcation. Thirty-five cortical branches were covered. A single FD stent was used in all patients. Symptomatic and asymptomatic periprocedural and delayed complications were reported. DSA and MRI controls were analyzed to evaluate modification of the aneurysm sac and jailed branches.
\end{abstract}

RESULTS Permanent morbidity was $3.4 \%$ (1/29), due to a jailed branch occlusion, with a modified Rankin Scale (mRS) score of 2 at the last follow-up. Mortality and permanent complication with poor prognosis (mRS score $>2$ ) rates were $0 \%$. The mean follow-up time for DSA and MRI (mean \pm SD) was $21 \pm 14.5$ months (range 3-66 months) and $19 \pm$ 16 months (range 3-41 months), respectively. The mean time to aneurysm sac occlusion (available for 24 patients), including stable remodeling, was $11.8 \pm 6$ months (median 13, range 3-27 months). The overall occlusion rate was $82.1 \%(23 / 28)$, and it was $91.7 \%$ (22/24) in the group of patients with at least 2 DSA control sequences. One recanalization occurred at 41 months posttreatment. At the time of publication, at the latest follow-up, 7 (20\%) of 35 covered branches were occluded, $18(51.4 \%)$ showed a decreased caliber, and the remaining $10(28.5 \%)$ were unchanged. MRI T2-weighted sequences showed complete sac reabsorption in 7/29 aneurysms (24.1\%), and the remaining lesions were either smaller (55.2\%) or unchanged (17.2\%). MRI revealed asymptomatic and symptomatic ischemic events in perforator territories in 7/28 (25\%) and 4/28 (14.3\%) patients, respectively, which were reversible within 24 hours.

CONCLUSIONS Flow diversion of bifurcation aneurysms is feasible, with low rates of permanent morbidity and mortality and high occlusion rates; however, recurrence may occur. Caliber reduction and asymptomatic occlusion of covered cortical branches as well as silent perforator stroke are common. Ischemic complications may occur with no identified predictable factors. MRI controls should be required in all patients to evaluate silent ischemic lesions and aneurysm sac reabsorption over time.

https://thejns.org/doi/abs/10.3171/2018.7.JNS18654

KEYWORDS intracranial aneurysm; endovascular treatment; flow diverter; recurrence; magnetic resonance imaging; vascular disorders; interventional neurosurgery

ABBREVIATIONS $A C O A=$ anterior communicating artery; $D S A=$ digital subtraction angiography; $D W I=$ diffusion-weighted imaging; $F D=$ flow diverter; $F R E D=$ flow redirection endoluminal device; $M C A=$ middle cerebral artery; $\mathrm{mRS}=$ modified Rankin Scale; $\mathrm{PED}=$ Pipeline embolization device; PerA = pericallosal artery; $\mathrm{SAH}=$ subarachnoid hemorrhage; TOF = time-of-flight; WEB = Woven EndoBridge.

SUBMITTED March 13, 2018. ACCEPTED July 19, 2018.

INCLUDE WHEN CITING Published online December 21, 2018; DOI: 10.3171/2018.7.JNS18654. 
$\mathrm{T}$ HE use of flow diverter (FD) stents has been largely approved for the treatment of carotid intracranial aneurysms. Recently, their use has been extended to include distal intracranial localizations. . $^{8,10,12,21,23} \mathrm{Nev}-$ ertheless, their use on bifurcation aneurysms beyond the circle of Willis-i.e., at the middle cerebral artery (MCA) bifurcation and at the anterior communicating artery (ACoA) complex-is still debated. ${ }^{4,26}$ Only a few articles with relatively small series and short follow-up times have been published, some with contradictory results. ${ }^{4,2121} \mathrm{~A}$ recently published meta-analysis on MCA aneurysms included 12 studies, of which only 8 were considered to be high quality. ${ }^{2}$ Only 1 study reports long-term followup based on clinical and digital subtraction angiography (DSA) imaging controls. ${ }^{14}$ MRI control data are also lacking. Gawlitza et al. previously published a series of 17 patients with a relatively short follow-up time (range 3-36 months), with MRI results, reporting on silent perforator stroke. ${ }^{12}$ The purpose of the current study is to present our series of consecutive patients and report procedure-related complications and medium-term anatomical and clinical results, with particular emphasis on aneurysm sac modifications on follow-up DSA and MRI sequences, and on the fate of cortical branches and perforating arteries when they are covered by a low-porosity device.

\section{Methods \\ Patient Population}

Between January 2010 and September 2017, 117 of 1549 patients from our center who underwent endovascular treatment for intracranial aneurysms were treated with FD stents. Among those 117 patients, 29 consecutive patients treated for reconstruction of complex aneurysms in the anterior circulation beyond the circle of Willis, i.e., at the MCA bifurcation and ACoA level, were the focus of our study. Prospectively maintained records of the patients were retrospectively evaluated. Preliminary short-term results of the first 18 aneurysms were published in a previous study. ${ }^{12}$

The primary outcome measure of our study was to determine complication rates of distal aneurysm FD treatment, including all consecutive patients from our series. Complications include asymptomatic and symptomatic events, intraprocedural, periprocedural ( $\leq 1$ week), and delayed ( $>1$ week), revealed either by clinical assessment or by imaging control with DSA and/or MRI.

Secondary outcome measures include the following: 1) DSA results: I) overall occlusion rate including all patients with at least 1 DSA control; II) medium-term results including all patients with $\geq 2$ DSA controls available; and III) analysis of covered branch modifications. 2) MRI results: I) presence of perforator strokes or any other procedure-related anomaly; and II) evaluation of sac reabsorption on $\mathrm{T} 2$ sequence.

\section{Treatment and Periprocedural Management}

Treatment indications were discussed and approved by a multidisciplinary neurovascular board. At our university hospital, endovascular treatment is considered the standard method for patients harboring unruptured or ruptured intracranial aneurysms, regardless of location. In our center, FD stents are not deployed during the acute phase of rupture. Consequently, FD stents are used in two situations: 1) nonacute ruptured aneurysms ( $>2-3$ weeks postrupture), or 2) unruptured aneurysms as the first or second line of treatment.

We can summarize our indications for the use of FD stents as follows: 1) recurrence after treatment with other techniques; 2) unsuitability of intrasaccular flow disrupters due to sac size ( $>10 \mathrm{~mm}$ ), previous coiling, or a partially thrombosed sac; 3) impossibility of performing $\mathrm{Y}$ stenting due to unfavorable morphology of the branches at the bifurcation; and 4) very large or giant aneurysms. In fact, lesions in the latter category show lower rates of immediate complete occlusion with conventional treatment methods and are prone to higher rates of recanalization and retreatment. ${ }^{15}$

Informed consent for the procedure and agreement to the use of anonymous clinical data in any possible retrospective studies were always obtained before the intervention. Patients were informed of all the risks, of all potential alternatives, and of the need for double antiplatelet therapy.

All procedures were performed after induction of general anesthesia. A biplane flat-panel DSA unit (Allura XperFD20, Philips) was used in a coaxial technique, in which a long 6-Fr introducer sheath was placed in the distal common carotid artery or the proximal internal carotid artery, an intermediate catheter (Fargomax, Balt; Sofia, Terumo MicroVention) was placed more distally in the internal carotid artery, and the appropriate FD microcatheter (Marksman, Covidien/ev3; Headway 27, MicroVention; Vasco 21, Balt) was advanced over suitable guidewires into the selected bifurcation branch. Special attention was paid to cover as few branches as possible when placing the FD stent. In aneurysms larger than 15 $\mathrm{mm}$, adjunctive coiling was performed through a jailed microcatheter after FD stent deployment. Femoral access was sealed with a closure device. Patients were extubated in the angiography suite and were admitted to a neurovascular intensive care unit for at least 24 hours.

All patients received double antiplatelet therapy 7 days prior to treatment and were screened for resistance against clopidogrel by applying platelet aggregometry testing and Verify Now testing on the day before the procedure. In the case of resistance, a double loading dose of clopidogrel $(600 \mathrm{mg})$ was immediately administered and the tests were repeated on the morning of the procedure. If the patient remained resistant to clopidogrel, the procedure was postponed and clopidogrel was switched to ticagrelor.

The intraprocedural heparin protocol included an intravenous bolus of $25 \mathrm{IU} / \mathrm{kg}$ and then continuous infusion with the intention to maintain an activated clotting time of approximately 2-3 times the baseline value. Heparin was stopped and reversed at the end of the procedure. Low-molecular-weight heparin at a preventive dosage was administered during the hospital stay. A 1-week postprocedure steroid treatment was given for aneurysms larger than $15 \mathrm{~mm}$.

According to our follow-up protocol, clopidogrel is stopped after 3 months and a DSA control is done approx- 
imately 2 weeks later. Acetylsalicylic acid is continued for another year, at a minimum.

\section{Follow-Up Protocol}

Our follow-up protocol consists of clinical evaluation, DSA, and MRI scheduled at 3-6 months and 12-18 months after treatment. Further DSA control sequences may be obtained depending on initial results. MRI controls are scheduled in conjunction with DSA controls or as the sole control every 12-24 months, even if DSA shows aneurysm sac occlusion. In our institution, all patients who undergo aneurysm embolization are followed up with MRI for 10-15 years after treatment, irrespective of embolization technique or treatment.

MRI was performed using a 3-T system (Achieva, Philips Medical Systems; Magnetom Skyra, Siemens AG). Our MRI protocol includes diffusion-weighted imaging (DWI), T2-weighted spin-echo imaging, and 3D time-offlight (TOF) sequences. T1-weighted postcontrast spinecho imaging was not performed routinely.

DWI comprised a multisection, single-shot, spin-echo echo planar imaging sequence. Diffusion gradients were applied in each of the $\mathrm{x}, \mathrm{y}$, and $\mathrm{z}$ directions with two $\mathrm{b}$ values $\left(0\right.$ and $\left.1000 \mathrm{sec} / \mathrm{mm}^{2}\right)$. For 3D TOF MR angiography: TE $3.69 \mathrm{msec}$; TR $22 \mathrm{msec}$; flip angle $20^{\circ}$; total acquisition time 6:32 minutes; number of sections 240; section thickness $0.52 \mathrm{~mm}$; rectangular field of view $190 \times 171$ $\mathrm{mm}$; acquisition matrix $320 \times 231$; reconstruction matrix $640 \times 462$; reconstructed voxel size $0.3 \times 0.3 \times 0.5 \mathrm{~mm}$; acquisition $0.74 \times 0.59 \times 0.104$.

\section{Image Analysis}

All patients' DSA images of the procedure as well as available DSA and MRI follow-up studies were reviewed by 2 readers, a neuroradiology fellow and an interventional neuroradiologist with more than 20 years of experience. In situations of discrepancy, a third investigator was used to validate an assessment (C.M., C.C., A.C.J.).

As previously published, immediate postprocedural and follow-up results of aneurysm sac filling and flow dynamics in the jailed arteries were analyzed. For immediate postprocedural results a variable 4-grade score, as described by Szikora et al., was used (grade 3, no change in aneurysm circulation; grade 2 , slight slowing of circulation without contrast medium stasis; grade 1, marked slowing with contrast medium stasis throughout the venous phase; or grade 0 , complete occlusion). ${ }^{25}$ Analysis of the flow dynamics in the jailed branch was graded as follows: normal antegrade flow (class 3, no change); slight decrease in velocity with sufficient persistent antegrade flow (class 2, slow flow); marked decrease in velocity with only a very slow antegrade flow (class 1 , very slow flow); and no flow (class 0 , occlusion). On follow-up DSA studies, the aneurysm sac was defined as occluded (class 0 ), decreased in size (class 1), or unchanged (class 2). Flow dynamics in jailed branches on follow-up DSA were categorized as normal (class 2), decreased in caliber compared to preoperative images (class 1), or occluded (class $0)$. Moreover, we applied the Cekirge-Saatci classification to define cases of stable remodeling, defined as class 5 oc- clusion for which at least 2 angiographic controls, at least 6 months apart, and expanding for a period of not less than 1 year, are required to demonstrate stability. 5,14

Follow-up MRI controls were reviewed and compared to pretreatment status and posttreatment DSA results to evaluate aneurysm sac modifications and shrinking of the external diameter of the aneurysm (i.e., perfused and thrombosed sac) on T2-weighted sequences, which is considered to be the definitive sign of embolization efficacy. Any ischemic lesions (symptomatic and/or asymptomatic) were noted, with attention given to the perforators and jailed branch territories.

All statistical analyses were performed using SPSS Statistics version 17 (SPSS, Inc.). The mean values are expressed \pm SD.

\section{Results}

\section{Population and Aneurysm Characteristics}

Detailed information regarding the patients, aneurysms, and stents are given in Table 1.

\section{Treatment and Technical Outcomes}

Two interventional neuroradiologists (C.C., A.C.J.) treated all patients with single FD stent coverage. The devices used included the Pipeline embolization device (PED; ev3/Covidien) in 15 patients; the flow redirection endoluminal device (FRED; MicroVention) in 12 patients; and the SILK flow diverter (SFD; Balt Extrusion) in 2 patients. No problems in device delivery were encountered. No oversizing of the FD stent was performed. Adjunctive use of coils due to the large size of the aneurysm was performed in 5 patients (17.2\%).

Thirty-five bifurcation branches were covered by FD stents. With regard to the MCA bifurcation, the upper middle cerebral branch was covered 14 times, the temporal branch 5 times, the posterior branch 4 times, and the frontal anterior branch 3 times. Concerning the ACoA region, an FD stent was placed in the homolateral $A_{1}-A_{2}$ segment covering the ACoA in 5 cases, whereas the left $\mathrm{A}_{2}$ was jailed in 3 cases, in which the FD stent was deployed from the left $A_{1}$ segment to the right $A_{2}$. The FD stent in the pericallosal artery (PerA) jailed the calloso-marginal artery.

\section{Complications}

Permanent morbidity was $3.4 \%(1 / 29)$ due to jailed branch occlusion, with a modified Rankin Scale (mRS) score of 2 at last follow-up. Mortality and permanent complication rate with poor prognosis $(\mathrm{mRS}$ score $>2$ ) were $0 \%$. Details of complications are shown in Table 2.

\section{Asymptomatic Complications}

Two intraprocedural asymptomatic complications were noted: 1 subtle subarachnoid hemorrhage (SAH), probably due to wire tip perforation of a distal MCA branch, with contrast medium collection on systematic postprocedure cone beam CT; and 1 intrastent acute partial thrombosis that resolved with high arterial pressure and pass-through of the stent with a microcatheter, which slightly modified the stent shape. 
TABLE 1. Patient, aneurysm, and stent characteristics

\begin{tabular}{|c|c|c|c|c|c|c|c|}
\hline $\begin{array}{l}\text { Case } \\
\text { No. }\end{array}$ & Presentation & $\begin{array}{l}\text { Aneurysm } \\
\text { No. }\end{array}$ & Side/Location & $\begin{array}{l}\text { Size of Neck/Dome } \\
(\mathrm{mm})\end{array}$ & $\begin{array}{l}\text { Dome-to- } \\
\text { Neck Ratio* }\end{array}$ & $\begin{array}{l}\text { FD Stent, Size } \\
\qquad(\mathrm{mm})\end{array}$ & $\begin{array}{l}\text { Additional } \\
\text { Coiling }\end{array}$ \\
\hline 1 & Headaches & 1 & Rt MCA & $9.4 / 6.5$ & 0.69 & SILK, $3.5 \times 25$ & No \\
\hline 2 & SAH 3 yrs prior-not treated & 2 & Rt MCA & $6.2 / 4.9$ & 0.79 & SILK, $3.5 \times 25$ & No \\
\hline 3 & $\begin{array}{l}\text { SAH, staged treatment w/ incomplete } \\
\text { coiling } 17 \text { days earlier }\end{array}$ & 3 & ACoA complex $†$ & $\begin{array}{l}\text { Total: } 2.1 / 3.1 \& 3.3 / 3.4 \\
\quad \text { residual: } 2.2 / 4.1\end{array}$ & 1.86 & PED, $2.5 \times 20$ & No \\
\hline 4 & $\begin{array}{l}\text { SAH, staged treatment w/ incomplete } \\
\text { coiling } 6 \text { mos earlier }\end{array}$ & 4 & Rt MCA & $\begin{array}{l}\text { Total: } 5.8 / 12.2 \\
\text { residual: } 5.8 / 9.4\end{array}$ & 1.62 & PED, $2.5 \times 20$ & No \\
\hline 5 & $\begin{array}{l}\text { SAH treated by coiling } 5 \text { yrs earlier, } \\
\text { now aneurysm recurrence }\end{array}$ & 5 & ACoA complex & $\begin{array}{l}\text { Total: } 4.6 / 9.5 \text {; residual: } \\
\quad 6.7 / 5.0\end{array}$ & 0.75 & PED, $2.5 \times 20$ & No \\
\hline 6 & $\begin{array}{l}\text { Increase in size of asymptomatic MCA } \\
\text { aneurysm; SAH w/ coiling of ACOA } \\
\text { aneurysm } 7 \text { yrs earlier }\end{array}$ & 6 & Rt MCA & $4.7 / 5.2$ & 1.10 & PED, $3.0 \times 12$ & No \\
\hline 7 & Headaches, vertigo & 7 & Rt MCA & $6.1 / 6.9$ & 1.13 & FRED, $3.5 \times 22 \times 16$ & No \\
\hline 8 & Asymptomatic & 8 & Rt MCA & $5.2 / 8.0$ & 1.54 & FRED, $3.5 \times 13 \times 7$ & No \\
\hline \multirow[t]{2}{*}{$9 \ddagger$} & Asymptomatic & 9 & Rt MCA & $3.3 / 5.4$ & 1.64 & PED, $2.5 \times 16$ & No \\
\hline & & 10 & Rt MCA & $4.0 / 6.9$ & 1.72 & & \\
\hline 10 & $\begin{array}{l}\text { SAH, staged treatment w/ incomplete } \\
\text { coiling } 10 \text { mos earlier }\end{array}$ & 11 & Lt MCA & $\begin{array}{l}\text { Total: 5.0/18; residual: } \\
\text { 5.0/6.0 }\end{array}$ & 1.20 & PED, $2.5 \times 16$ & No \\
\hline 11 & $\begin{array}{l}\text { SAH, staged treatment w/ very incom- } \\
\text { plete coiling } 7 \text { mos earlier }\end{array}$ & 12 & ACoA complex & $\begin{array}{l}\text { Total: 4.0/7.0; residual: } \\
\text { 4.0/4.0 }\end{array}$ & 1.00 & PED, $2.5 \times 16$ & No \\
\hline 12 & $\begin{array}{l}\text { Headaches; recanalized aneurysm } \\
\text { after treatment by intrasaccular flow } \\
\text { disruption (WEB) } 6 \text { mos earlier }\end{array}$ & 13 & Rt MCA & $\begin{array}{l}\text { Total: } 8.8 / 10.4 \\
\text { residual: } 8.8 / 5.3\end{array}$ & 0.60 & FRED, $3.5 \times 22 \times 16$ & No \\
\hline 13 & $\begin{array}{l}\text { Visual problems due to compressive ef- } \\
\text { fect of a giant, partially thrombosed } \\
\text { aneurysm on the optic chiasm }\end{array}$ & 14 & ACoA complex & $6.2 / 18.5$ & 2.98 & FRED, $3.5 \times 22 \times 16$ & Yes \\
\hline 14 & $\begin{array}{l}\text { SAH, staged treatment w/ incomplete } \\
\text { coiling } 7 \text { mos earlier }\end{array}$ & 15 & Rt MCA & $\begin{array}{l}\text { Total: NA/14.0; } \\
\text { residual: NA/6.0 }\end{array}$ & NA§ & PED, $2.5 \times 12$ & No \\
\hline 15 & Asymptomatic & 16 & ACoA complex & $5.2 / 8.0$ & 1.54 & PED, $2.5 \times 16$ & No \\
\hline 16 & $\begin{array}{l}\text { SAH treated by coiling } 10 \text { yrs before, } \\
\text { now aneurysm recurrence }\end{array}$ & 17 & Lt MCA & $\begin{array}{l}\text { Total: } 7.3 / 5.2 \text {; residual: } \\
\quad 4.0 / 4.0\end{array}$ & 1.00 & FRED, $3.5 \times 13 \times 7$ & No \\
\hline 17 & $\begin{array}{l}\text { SAH treated by coiling } 3 \text { yrs earlier, } \\
\text { now aneurysm recurrence }\end{array}$ & 18 & Lt MCA & $\begin{array}{l}\text { Total: } 5.0 / 10.0 \\
\text { residual: } 8.0 / 8.0\end{array}$ & 1.00 & PED, $2.5 \times 16$ & No \\
\hline 18 & Asymptomatic & 19 & ACoA complex & $5.7 / 22.0$ & 3.85 & FRED, $3.5 \times 13 \times 7$ & Yes \\
\hline 19 & Epilepsy & 20 & ACoA complex & $5.5 / 6.6$ & 1.20 & FRED, $3.5 \times 11 \times 7$ & No \\
\hline 20 & Asymptomatic & 21 & ACoA complex & $8.9 / 12.1$ & 1.36 & FRED, $3.5 \times 22 \times 16$ & No \\
\hline 21 & Asymptomatic & 22 & Lt MCA & $6.3 / 10.2$ & 1.62 & FRED, $3.5 \times 22 \times 16$ & No \\
\hline 22 & Asymptomatic & 23 & PerA & $4.7 / 8.0$ & 1.70 & PED, $2.5 \times 14$ & No \\
\hline 23 & Asymptomatic & 24 & Rt MCA & $7.2 / 13$ & 1.81 & PED, $3 \times 14$ & No \\
\hline 24 & Epilepsy & 25 & Rt MCA & $3.2 / 27$ & 8.40 & PED, $2.5 \times 14$ & Yes \\
\hline 25 & $\begin{array}{l}\text { Asymptomatic; treated by coiling in } \\
2002, \text { now aneurysm recurrence }\end{array}$ & 26 & Lt MCA & $\begin{array}{l}\text { Total: NA/NA; residual: } \\
\text { 8.0/NA }\end{array}$ & NA & PED, $3 \times 14$ & No \\
\hline 26 & $\begin{array}{l}\text { Asymptomatic; treated by coiling in } \\
2013, \text { now aneurysm recurrence }\end{array}$ & 27 & Lt MCA & $\begin{array}{l}\text { Total: } 5.6 / 13 \text {; residual: } \\
\text { 5.6/8.2 }\end{array}$ & 1.57 & PED flex, $3 \times 14$ & No \\
\hline 27 & Asymptomatic & 28 & Rt MCA & $5.2 / 22$ & 4.23 & FRED, $3 \times 27 \times 21$ & Yes \\
\hline 28 & $\begin{array}{l}\text { Asymptomatic; treated by coiling in } \\
2008, \text { now aneurysm recurrence }\end{array}$ & 29 & Rt MCA & $\begin{array}{l}\text { Total: } 5.0 / 13.0 \\
\text { residual: } 5.0 / 8.0\end{array}$ & 1.6 & $\begin{array}{l}\text { FRED Jr, } 3 \times 27 \\
\quad \times 21\end{array}$ & No \\
\hline 29 & $\begin{array}{l}\text { Asymptomatic \& growing on FU since } \\
2010\end{array}$ & 30 & Lt MCA & $8.0 / 16.0$ & 2.00 & FRED mini, $2.5 \times 18$ & Yes \\
\hline
\end{tabular}




\section{» CONTINUED FROM PAGE 1754}

\section{TABLE 1. Patient, aneurysm, and stent characteristics}

\begin{tabular}{|c|c|c|c|c|c|c|c|}
\hline $\begin{array}{l}\text { Case } \\
\text { No. }\end{array}$ & Presentation & $\begin{array}{c}\text { Aneurysm } \\
\text { No. }\end{array}$ & Side/Location & $\begin{array}{l}\text { Size of Neck/Dome } \\
(\mathrm{mm})\end{array}$ & $\begin{array}{l}\text { Dome-to- } \\
\text { Neck Ratio* }\end{array}$ & $\begin{array}{l}\text { FD Stent, Size } \\
(\mathrm{mm})\end{array}$ & $\begin{array}{c}\text { Additional } \\
\text { Coiling }\end{array}$ \\
\hline
\end{tabular}

FU = follow-up; NA = not available.

The table shows data of 29 consecutive patients (15 men, 14 women; mean age 54.7 years, range 39-69 years) harboring 30 aneurysms: 21 were located at the MCA bifurcation (14 right, 7 left), 8 at the ACoA region, and 1 at the PerA bifurcation. All aneurysms were either unruptured, a recurrence, or undergoing delayed staged treatment of a ruptured aneurysm. Twenty patients presented with unruptured aneurysm that was incidentally discovered $(n=17)$ or symptomatic $(n=3 ;$ compressive syndrome, epilepsy). Nine patients had a previous SAH; 8 were treated and 1 was left untreated 3 years prior. Fifteen were small aneurysms $(<10 \mathrm{~mm}), 11$ were large $(10-25 \mathrm{~mm})$, and 4 were giant $(>25 \mathrm{~mm})$, according to Yasargil's classification, with a mean dome-to-diameter ratio of $10.8 \pm 10.0$ (range 4-27). The dome-to-neck ratio was $2.2 \pm 3.2$ (median 1.5). Twelve aneurysms (40\%) were residual sac or recurrence in a patient previously treated by either coiling $(n=11)$ or endosaccular flow disruption (WEB device, $n=1$ ). The mean diameter of residual sac in this group was $6.1 \pm 1.9 \mathrm{~mm}$ (range $4-9.4 \mathrm{~mm}$ ). The mean diameter of previously untreated aneurysms was $11.8 \pm 6.9 \mathrm{~mm}$.

* Dome-to-neck ratio of the remnant/recurrence is displayed.

$\dagger$ Aneurysm consisting of 2 sacs, of which only 1 could be coiled. The other sac increased in size during follow-up.

$\ddagger$ Two separate aneurysms.

$\S$ Not available in this aneurysm without a defined neck.

TABLE 2. Periprocedural and delayed complications

\begin{tabular}{|c|c|c|c|c|}
\hline \multirow[b]{2}{*}{ Case No. } & \multicolumn{2}{|c|}{ Periprocedural Comps ( $\leq 1$ st wk) } & \multicolumn{2}{|c|}{ Delayed Comps (>1st wk) } \\
\hline & Asymptomatic & Symptomatic & Asymptomatic & Symptomatic \\
\hline 4 & None & None & $\begin{array}{l}\text { Silent perforator stroke } \\
\text { after } 9 \text { mos }\end{array}$ & None \\
\hline 7 & None & None & $\begin{array}{l}\text { Silent perforator stroke } \\
\text { after } 4 \text { mos }\end{array}$ & None \\
\hline 8 & None & None & $\begin{array}{l}\text { Silent perforator stroke } \\
\text { after } 4 \text { mos }\end{array}$ & $\begin{array}{l}\text { New perforator stroke after } 6 \text { mos: tran- } \\
\text { sient ( } 20 \text { mins) hemihypesthesia }\end{array}$ \\
\hline 9 & None & None & $\begin{array}{l}\text { Minimal sulcal hemor- } \\
\text { rhage (unknown origin) }\end{array}$ & None \\
\hline 10 & None & None & None & $\begin{array}{l}\text { Perforator stroke after } 17 \text { days: hemihypes- } \\
\text { thesia, completely reversible after } 24 \text { hrs }\end{array}$ \\
\hline 13 & None & $\begin{array}{l}\text { Heubner's perforator stroke after } 3 \\
\text { days: rt facial paresis, completely } \\
\text { reversible after } 24 \mathrm{hrs}\end{array}$ & None & None \\
\hline 14 & $\begin{array}{l}\text { Sulcal SAH due to } \\
\text { wire tip perforation }\end{array}$ & None & None & None \\
\hline 15 & None & None & $\begin{array}{l}\text { Silent Heubner's perfora- } \\
\text { tor stroke after } 4 \text { mos }\end{array}$ & None \\
\hline 17 & None & None & $\begin{array}{l}\text { Silent perforator stroke } \\
\text { after } 4 \text { mos }\end{array}$ & None \\
\hline 19 & $\begin{array}{l}\text { Intrastent partial } \\
\text { thrombosis }\end{array}$ & None & $\begin{array}{l}\text { Silent ischemic lesions } \\
\text { after } 4 \text { mos }\end{array}$ & None \\
\hline 21 & None & $\begin{array}{l}\text { Immediate branch occlusion \& perfora- } \\
\text { tor stroke: Broca's aphasia \& rt } \\
\text { hemiparesis; mRS score } 2 \text { at last FU }\end{array}$ & None & None \\
\hline 24 & None & $\begin{array}{l}\text { Postop hemiplegia after treatment, } \\
\text { completely reversible after } 1 \mathrm{hr} \text {, } \\
\text { normal DSA, probable mass effect }\end{array}$ & $N A^{*}$ & None \\
\hline 28 & None & None & $\begin{array}{l}\text { Silent ischemic lesions } \\
\text { after } 4 \text { mos }\end{array}$ & None \\
\hline Comp rate & $6.9 \%(2 / 29)$ & $10.3 \%(3 / 29)$ & $28.5 \%(8 / 28)$ & $6.9 \%(2 / 29)$ \\
\hline
\end{tabular}

Comp = complication.

* For the patient in case 24 no MRI was available; follow-up by phone call interview at 14 months after embolization. 


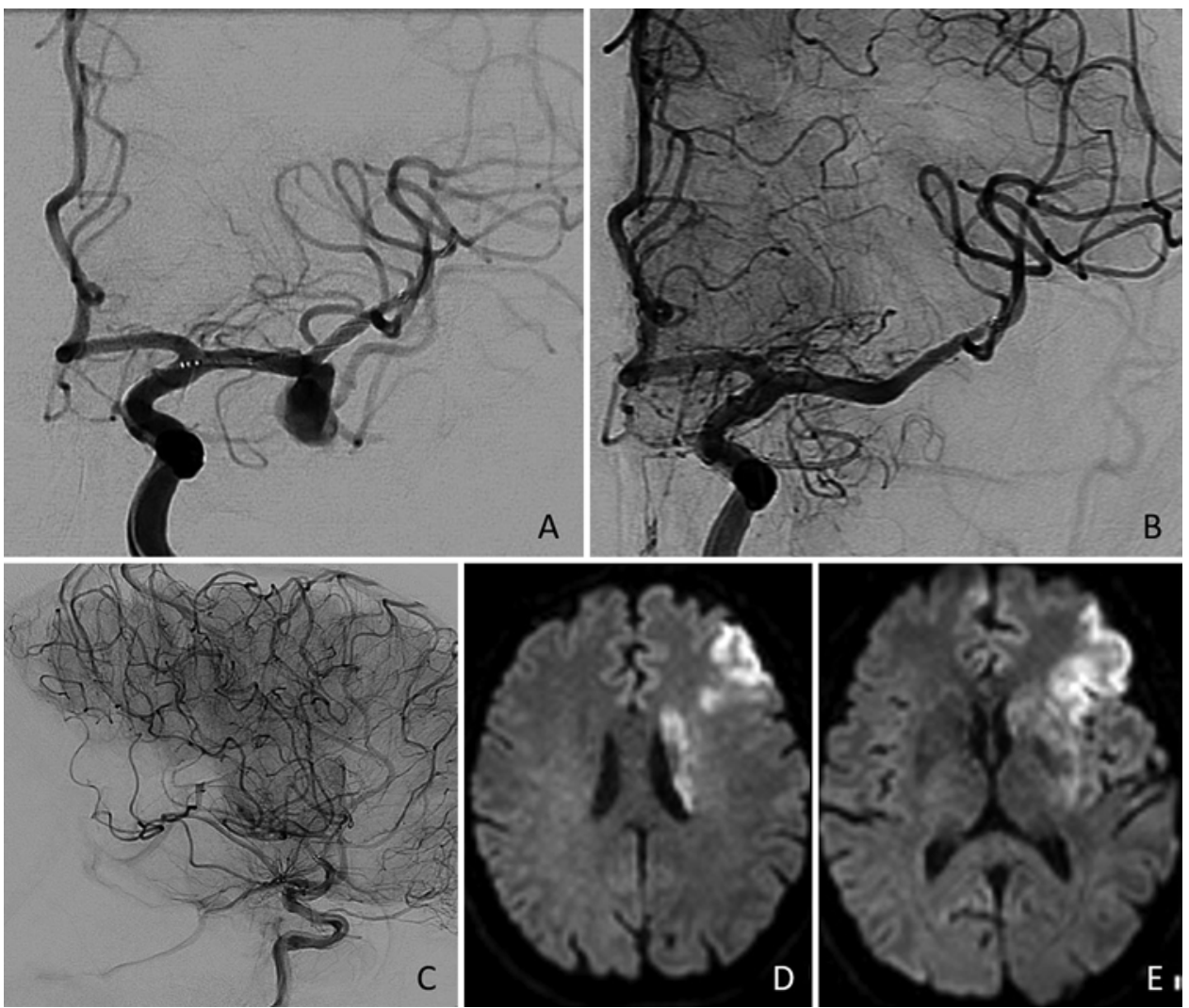

FIG. 1. DSA control during deployment of the FD stent in the upper MCA branch and $M_{1}$, covering $M_{1}$ perforators, the inferior branch coming from the aneurysm sac, and 2 anterior branches originating from the upper branch (A). Immediate control after deployment shows acute occlusion of the aneurysm sac and of the inferior branch, as well as of one of the anterior branches (B and $\mathbf{C})$. Immediate MRI control sequences show ischemic lesions of the lenticulostriate and frontoinsular and temporal territories (D and E). Patient presented with Broca's aphasia and right hemiparesis.

Delayed asymptomatic perforator stroke was observed on follow-up MRI in 7 patients (as described in MRI Findings).

\section{Symptomatic Complications}

Symptomatic complications occurred in 5 patients (17.2\%)-3 periprocedural ( $\leq 1$ week) and 2 delayed. Two periprocedural symptomatic complications were transient: 1 Heubner's perforator stroke resolved in $<24$ hours and 1 hemiplegia resolved in 1 hour. The latter complication was probably due to increased mass effect in a patient harboring a giant aneurysm (no branch or stent occlusion, nor an ischemic lesion on MRI). The third patient presented with postprocedure aphasia and hemiparesis due to an intraprocedural jailed branch occlusion (Fig. 1). This patient finally recovered almost completely, with an $\mathrm{mRS}$ score of 2 at 16-months follow-up.

Symptomatic delayed complications were observed in 2 patients. One patient presented with sudden onset of hemihypesthesia that lasted for 24 hours, 17 days after treatment, and the second patient had 20 minutes of hemihypesthesia, which occurred 6 months after treatment. Both cases showed stroke of a lenticulostriate artery covered by the FD stent, diagnosed on the acute-phase MRI.

All patients who had ischemic events or transient symptoms were clopidogrel responders, and no patient needed a double dose before treatment. The patient in case 21, however, happened to be highly responsive to clopidogrel, showing values of 7-9 platelet reactivity units (PRUs).

Absence of aneurysm rupture and $0 \%$ procedure-related mortality were confirmed for the population throughout the follow-up period. One patient died of unrelated causes (pulmonary carcinoma).

\section{Aneurysm Occlusion Rates and Covered Branch Modification}

Anatomical immediate and follow-up results are summarized in Table 3. Immediately after implantation of the device, an occlusion was observed in 3 of 30 aneurysms (10\%; cases 3,13 , and 21). In 1 case the lesion remained occluded over time, 1 case showed a residual aneurysm at last follow-up, and in the third case the aneurysm was occluded at 27 months. Three aneurysms showed stasis after FD stent placement, 16 presented slow flow in the sac, and 8 were unchanged.

\section{Short-Term Follow-Up DSA Results}

Short-term (3-6 months) follow-up DSA results were available for 27 patients. Two patients have not received the first DSA control yet. Four patients were recently treated and have not received a second control. One patient died 
TABLE 3. Immediate and latest follow-up results

\begin{tabular}{|c|c|c|c|c|c|c|c|c|c|c|}
\hline \multirow[b]{2}{*}{ Case No. } & \multirow[b]{2}{*}{$\begin{array}{c}\text { Aneurysm } \\
\text { No. }\end{array}$} & \multicolumn{3}{|c|}{ Immediate Results } & \multicolumn{6}{|c|}{ Last FU Results } \\
\hline & & $\begin{array}{l}\text { Aneurysm } \\
\text { Sac Score* }\end{array}$ & $\begin{array}{c}\text { Jailed } \\
\text { Artery(ies) }\end{array}$ & $\begin{array}{c}\text { Jailed } \\
\text { Branch } †\end{array}$ & $\begin{array}{l}\text { Last DSA } \\
\text { (mos) }\end{array}$ & $\begin{array}{l}\text { Time to Sac Occl } \\
\text { or Stability (mos) }\end{array}$ & $\begin{array}{c}\text { Sac } \\
\text { DSA }\end{array}$ & $\begin{array}{l}\text { Jailed } \\
\text { Branch§ }\end{array}$ & $\begin{array}{l}\text { Last MRI } \\
\text { (mos) }\end{array}$ & $\begin{array}{l}\text { Sac } \\
\text { MRIf }\end{array}$ \\
\hline 1 & 1 & 3 & uMCA, tMCA & 3,3 & 59 & 13 & 0 & 2,2 & 13 & 1 \\
\hline 2 & 2 & 3 & uMCA, tMCA & 2,2 & 66 & 8 & 0 & 2,2 & 35 & 1 \\
\hline 3 & 3 & 0 & Lt $\mathrm{A}_{2}$ & 0 & 15 & 3 & 0 & 2 & 15 & 0 \\
\hline 4 & 4 & 3 & pMCA, tMCA, aMCA & $3,3,0$ & 27 & 27 & 0 & $2,2,1$ & 27 & 0 \\
\hline 5 & 5 & 2 & $\mathrm{ACoA}$ & 3 & 17 & 5 & 0 & 0 & 33 & 1 \\
\hline 6 & 6 & 2 & aMCA & 3 & 41 & 17 & $\operatorname{Rec}$ & 1 & 41 & 1 \\
\hline 7 & 7 & 2 & UMCA & 3 & 17 & 4 & 0 & 2 & 17 & 1 \\
\hline 8 & 8 & 3 & UMCA & 3 & 15 & 15 & 0 & 1 & 12 & 2 \\
\hline \multirow[t]{2}{*}{9} & 9 & 2 & UMCA & 3 & 16 & 4 & 0 & 1 & 16 & 0 \\
\hline & 10 & 2 & & & 16 & 16 & 0 & & & 0 \\
\hline 10 & 11 & 3 & UMCA & 2 & 28 & 3 & 0 & 1 & 28 & 0 \\
\hline 11 & 12 & 2 & Lt $\mathrm{A}_{2}$ & 0 & 13 & 3 & 0 & 0 & 13 & 0 \\
\hline 12 & 13 & 3 & UMCA & 3 & 25 & 12 (stable) & 1 & 1 & 37 & 1 \\
\hline 13 & 14 & 0 & Lt $\mathrm{A}_{2}$ & 3 & 14 & 4 (stable) & 1 & 1 & 26 & 1 \\
\hline 14 & 15 & 2 & pMCA & 1 & 15 & 15 & 0 & 1 & 4 & 2 \\
\hline 15 & 16 & 1 & $\mathrm{ACoA}$ & 3 & 16 & 16 & 0 & 2 & 32 & 1 \\
\hline 16 & 17 & 2 & tMCA & 3 & 39 & 27 & 0 & 0 & 39 & 1 \\
\hline 17 & 18 & 2 & UMCA & 3 & 16 & 16 & 0 & 1 & 28 & 1 \\
\hline 18 & 19 & 2 & $\mathrm{ACoA}$ & 3 & 14 & 14 & 0 & 0 & 14 & 2 \\
\hline 19 & 20 & 2 & $\mathrm{ACoA}$ & 2 & 13 & 4 & 0 & 0 & 13 & 1 \\
\hline 20 & 21 & 2 & $\mathrm{ACoA}$ & 2 & 4 & Death & 1 & 2 & 4 & 1 \\
\hline 21 & 22 & 0 & pMCA, tMCA, aMCA & $0,0,0$ & 27 & 27 & 0 & $1,1,0$ & 15 & 1 \\
\hline 22 & 23 & 2 & PerA & 3 & 17 & 4 & 0 & 0 & 17 & 0 \\
\hline 23 & 24 & 2 & UMCA & 3 & 16 & 16 & 0 & 1 & 16 & 1 \\
\hline 24 & 25 & 1 & UMCA & 2 & - & - & - & - & - & NA \\
\hline 25 & 26 & 3 & UMCA & 3 & 18 & 6 & 0 & 1 & 15 & 0 \\
\hline 26 & 27 & 2 & UMCA & 2 & 5 & - & 1 & 1 & 5 & 1 \\
\hline 27 & 28 & 2 & UMCA & 3 & 3 & - & 1 & 1 & 3 & 2 \\
\hline 28 & 29 & 3 & UMCA & 3 & 15 & 15 & 0 & 1 & 15 & 1 \\
\hline 29 & 30 & 1 & pMCA & 2 & - & - & - & Patent on MRI & 3 & 2 \\
\hline Mean \pm SD & & & & & $21 \pm 14.5$ & $11.8 \pm 6$ & & & $19 \pm 16$ & \\
\hline Range & & & & & $3-66$ & $3-27$ & & & $3-41$ & \\
\hline
\end{tabular}

aMCA = anterior branch of the MCA; Occl = occlusion; $\mathrm{PMCA}=$ posterior branch of the MCA; Rec = recurrence; tMCA = temporal branch of the MCA; uMCA = upper branch of the MCA; $-=$ no follow-up available yet.

Immediate occlusion rate was $10.7 \%$. Classifications were made according to Szikora et al.

* 3 = no change; 2 = slower circulation but no stasis; 1 = slower circulation with contrast medium stasis; $0=$ occlusion —complete stagnation without further contrast flow into the aneurysm.

$\dagger 3$ = no change in flow; 2 = slow flow; 1 = very slow flow; $0=$ occlusion.

$\ddagger 2=$ no change $; 1=$ decreased diameter; $0=$ occlusion.

$\S 2=$ normal caliber and patent; $1=$ decreased caliber but patent; $0=$ occluded.

before the second DSA control due to an unrelated cause (pulmonary carcinoma). DSA control at 12-18 months was available for 24 aneurysms. For 18 patients, DSA controls were stopped at complete aneurysm sac occlusion or after 2 controls demonstrated stability at more than 1 year.

The overall mean follow-up time for DSA was $21 \pm 14.5$ months (range 3-66 months). From a 10\% aneurysm occlusion rate on the immediate postprocedure angiograms, the series progressed to an overall occlusion rate of $82.1 \%$
(23/28), including patients with only 1 short-term followup DSA control. However, considering only those patients who underwent at least 2 DSA controls, 22/24 aneurysms were either totally occluded (20 cases) or had stable remodeled regional angioarchitecture, as described in the Cekirge-Saatci classification (2 cases), with an occlusion rate of $91.7 \%$ (Fig. 2).

For each patient the time to occlusion was calculated. The mean time to sac occlusion available for 24 patients, 


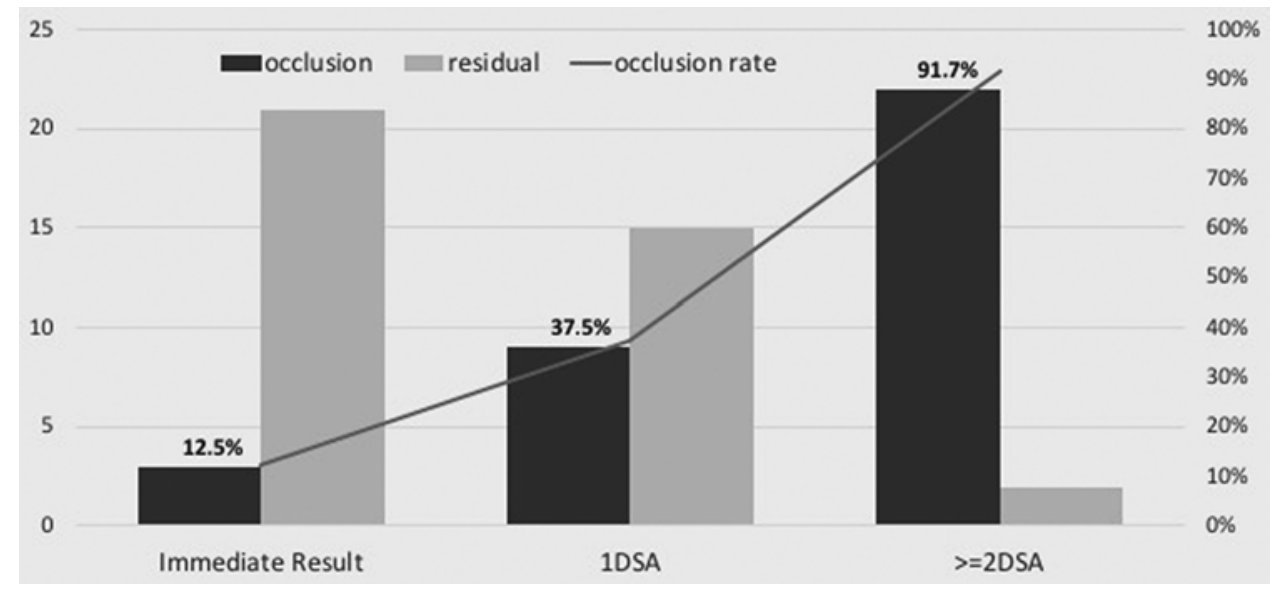

FIG. 2. Progressive occlusion at follow-up. Occlusion rate in the group of 24 patients with at least 2 DSA controls.

including those with class 5 Cekirge-Saatci classification, was $11.8 \pm 6$ months (median 13, range 3-27 months). However, 1 late recurrence with a small recirculation at the aneurysm neck appeared over time at 41 months, in a patient with an occlusion confirmed at the 17-month DSA control (case 6) (Fig. 3). The last follow-up results for each patient are depicted in the graph in Fig. 4.

\section{Covered Bifurcation Branches}

Thirty-five branches were covered by FD stents. Six jailed bifurcation branches were occluded immediately after FD stent delivery (17.1\%), whereas 9 branches (25.7\%) showed a slowed blood flow and 20 branches (57.1\%) were unchanged. At the latest follow-up, 7 covered branches were occluded (20\%); 18 (51.4\%) showed a decreased caliber, 7 of which showed some retrograde filling; and the remaining $28.5 \%$ (10/35) were unchanged. Remarkably, we observed that modification of the branches' caliber may not be stable over time: branches may reduce in caliber and then return. We had 3 cases in which the branch came back to the original caliber at long term. In the single case of recurrence, the jailed branch almost disappeared and the aneurysm was occluded at 17 months, but then at 41 months the branch was reperfused and the aneurysm neck recanalized (Fig. 3).

\section{MRI Findings}

MRI control was available for 29 aneurysms in 28 patients. The mean follow-up time was $19 \pm 16$ months (range 3-41 months). The last follow-up MRI sequence was compared to pretreatment MRI and to DSA results.

\section{Ischemic Lesions}

Eleven patients (39.3\%) presented with lesions-both symptomatic and asymptomatic-in perforating artery territories that were covered by an FD stent. The first follow-up MRI sequence revealed a silent perforator stroke in 7 of 28 patients (25\%); the other 4 patients $(14.3 \%)$ were symptomatic either in the early postprocedural phase (n $=2$ ) or in a delayed fashion $(n=2)$. All symptomatic perforator strokes were reversible within 24 hours. The MRI study showed perforators and cortical stroke in the territory of the occluded frontal jailed branch in only 1 case (Fig. 1).

\section{Aneurysm Sac Modifications}

MRI T2-weighted sequences showed complete sac reabsorption of $7 / 29$ aneurysms (24.1\%) and sac reduction of 16/29 aneurysms (55.2\%); the remaining 5/29 were unchanged (17.2\%). Of the 20 aneurysms with proven occlusion on DSA and available MRI control, 7 presented with complete sac reabsorption on T2 sequences on last MRI follow-up. The remaining aneurysms were either smaller $(n=10)$ or unchanged $(n=3)$. Two aneurysms with stable remodeling were smaller on MRI. Six aneurysms, still perfused on DSA, presented with either reduction $(n=4)$ or stability $(n=2)$ of the aneurysm sac on T2 sequences (Fig. 5).

\section{Discussion}

The choice between surgical and endovascular management of complex MCA and anterior cerebral artery aneurysms is still a matter of debate. ${ }^{10,22}$ Conventional microsurgical and endovascular techniques such as clipping and coil embolization with or without assistance (balloon or stent) are the mainstay for the treatment of such aneurysms. However, bifurcation aneurysms are often wide-necked, incorporate 1 or more side-branch vessels, and tend to have an unfavorable anatomical configuration. Moreover, aneurysms that recur after clipping or coiling pose significant technical challenges for conventional microsurgical and endovascular treatments.

For more than 5 years the off-label use of FD stents for the treatment of distal aneurysms has been reported, ${ }^{6,21}$ but remains debated. Several endovascular techniques, such as stent-assisted coiling, ${ }^{28}$ balloon remodeling, ${ }^{9} \mathrm{Y}$ stenting with or without coiling, ${ }^{24}$ and Woven EndoBridge (WEB) device, ${ }^{20}$ have been used for the treatment of bifurcation aneurysms, but results remain suboptimal with significant recurrence and complication rates. Several reports have demonstrated that the treatment of these types of aneurysms is feasible and safe, , $^{8,10,12,14,19,21,23,29}$ whereas others 


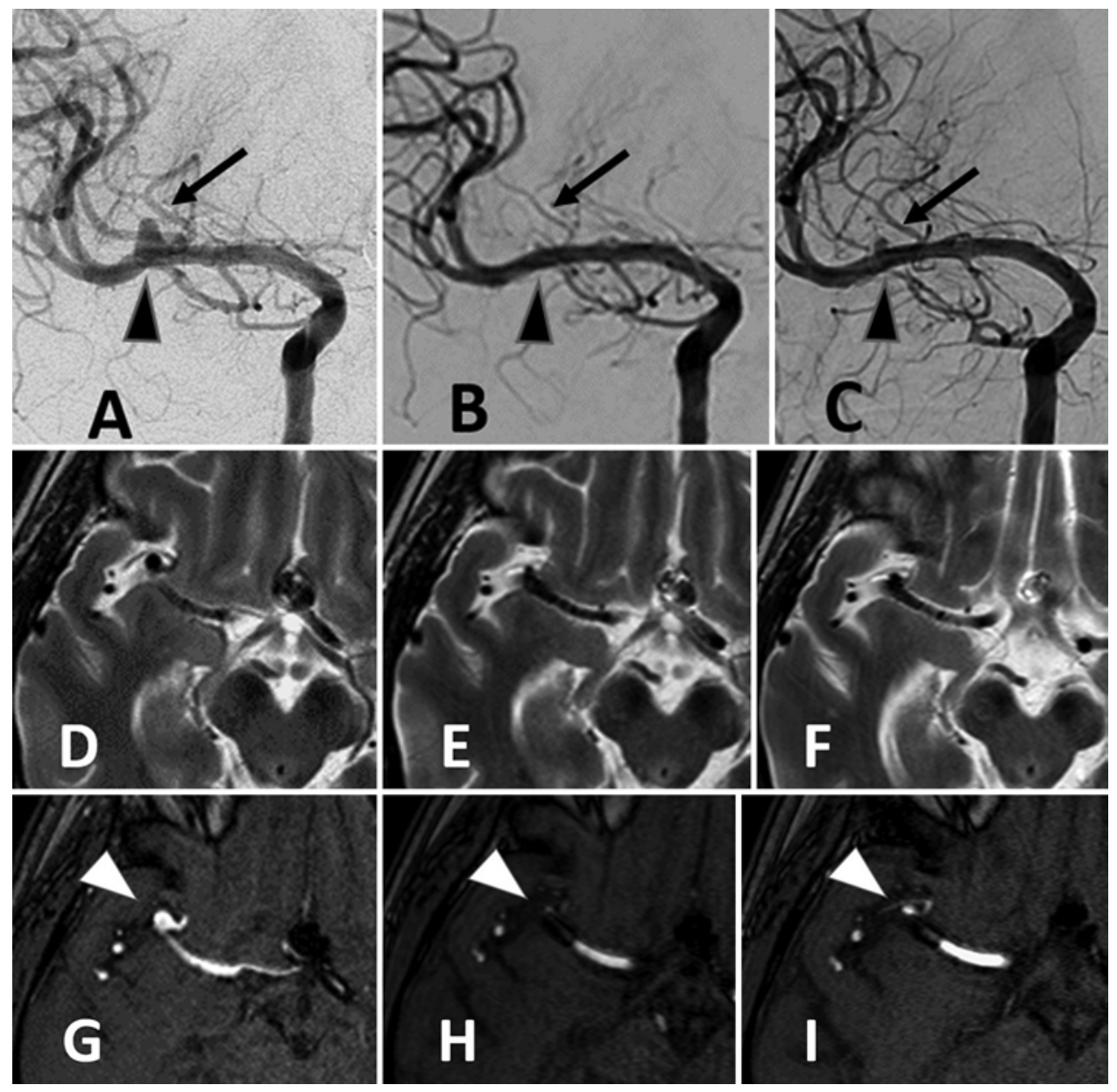

FIG. 3. A middle-aged patient with previous SAH due to ACoA aneurysm rupture treated by coiling presented with a small asymptomatic MCA aneurysm. He was treated by placing a PED FD covering the anterior branch. A: Baseline angiogram showing MCA aneurysm (arrowhead) and the anterior branch (arrow). B: DSA control obtained at 17 months showed aneurysm occlusion (arrowhead) and significant reduction in caliber of the anterior branch, which had almost disappeared (arrow). C: DSA control obtained at 41 months shows recirculation in both the arterial branch (arrow) and the aneurysm (arrowhead), with small recurrence at the neck. D and G: Baseline MRI controls with T2 sequences and 3D TOF before treatment. E and H: MRI controls obtained at 17 months showing complete disappearance of the aneurysm on both T2 and TOF sequences (arrowhead). Associated remodeling of the anterior branch is better observed on DSA (panel B). F and I: Latest follow-up after 41 months showing that recanalization has occurred with good correlation between DSA and 3D TOF images (arrowhead).

have shown morbidity and mortality rates rising up to $21 \%$ and $10 \%$, respectively. ${ }^{4,26}$

The most common complications are periprocedural thrombus formations, arterial branch perforation, in-stent thrombosis, side-branch occlusion, and perforator strokes. We reported at least 1 of each, yet they were asymptomatic or completely reversible by $<24$ hours. In only 1 case, we observed a symptomatic permanent stroke related to the jailing of cortical branches, which explains our morbidity rate of $3.4 \%$.

There was no mortality, which in the literature is usually related to delayed hemorrhagic complications. Even though the overall morbidity remained acceptable and in accordance with previous studies, the asymptomatic and reversible complications rate was surprisingly high. Perforator stroke occurred in more than one-third of the patients (39.3\%), either silent (25\%) or symptomatic but reversible
(14.3\%). These results confirm the preliminary results published in the study by Gawlitza et al., ${ }^{12}$ which was, to our knowledge, the first publication systematically reporting the presence of symptomatic and asymptomatic perforator stroke on routine MR control after a single FD stent was placed in a distal artery. Previous authors reported isolated, symptomatic cases, in series without systematic MRI control, in which 1 or more devices were used. ${ }^{11,16,18,27}$

The major prevalence of stroke in the perforator territory compared to the cortical branches when covered by an FD stent has already been discussed in our previous publication. ${ }^{12}$ As described by Iosif and colleagues, jailed cortical branches may find a new hemodynamic balance with leptomeningeal anastomosis, and fluctuate over time, maintaining some laminar direct perfusion through the stent with continuous changes in caliber. ${ }^{13,14}$ However, complications may occur in the acute phase. In our case, it 


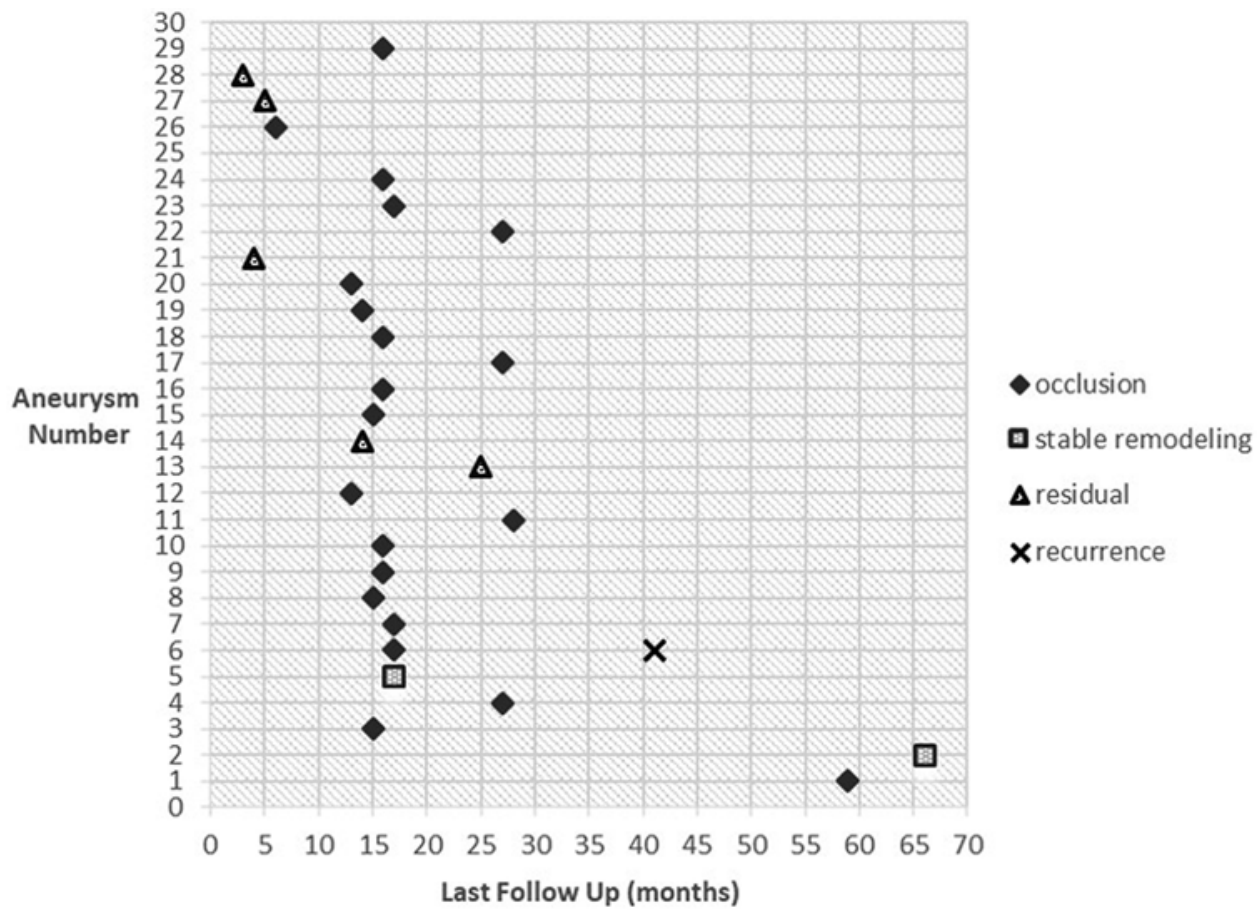

FIG. 4. Last follow-up DSA results for each aneurysm over time (months). Follow-up time is given on the x-axis; individual aneurysms are listed on the y-axis. Different shapes are used to indicate last follow-up results: occlusion (black rhombus), stable remodeling (gray square), residual aneurysm (triangle), recurrence (cross). Most of the patients showed occlusion within the second DSA control at 13-17 months. Two aneurysms had a persisting residual sac even after 2 DSA controls (cases 13 and 14). The patient in case 6 had a late recurrence at 41 months.

could be explained by the fact that the FD stent deployed in the upper branch was too long and covered too many branches (i.e., the anterior, temporal, and lower branch), so this might have prevented a new hemodynamically effective balance between leptomeningeal and direct perfusion. Moreover, the ischemic lesions were mostly in the anterior and temporal region, where smaller branches were jailed, whereas the acute occlusion of the lower branch caused no cortical ischemic sequelae, and only a few areas of DWI hyperintensity on postoperative MRI (Fig. 1).

\section{Occlusion Rate, Sac Reabsorption, and Recurrence}

Most of the reported populations are small and either present combined locations, at and beyond the circle of Willis, or focalize on MCA or the anterior cerebral artery and ACoA region. In our study we included a heterogeneous group of bifurcation aneurysms beyond the circle of Willis-21 MCA, 8 ACoA, and 1 PerA. We report an occlusion rate of $37.5 \%$ at first DSA control, rising to $91.7 \%$ at long-term follow-up (i.e., with $>2$ DSA controls). This slow pattern of occlusion is described in other studies ${ }^{8,14}$ with high occlusion rates (> 80\% at long-term follow-up), suggesting that occlusion could be a matter of time. The mean time of occlusion was 11.6 months, with a wide range from 3 to 27 months. Moreover, our analysis of immediate sac flow changes did not show an association with long-term results.

Occlusion predictor factors are difficult to identify. Some authors have analyzed the anatomy of MCA bifur- cation, distinguishing aneurysms located on a "true bifurcation" (classic bifurcation of MCA into superior and inferior trunks) or on a "variant bifurcation" (bifurcation of "early" anterior frontal or "early/distal" temporal branches), and have reported lower occlusion rates for true bifurcation compared to variant bifurcation, with a strong association between sac and side-branch occlusion. ${ }^{26}$ In our study, no consistent speculation may be done on this subject. We had only 2 cases of noncomplete occlusion: 1 large MCA aneurysm with a recurrence after a WEB device was placed, and 1 giant aneurysm of the ACoA region. In both cases the aneurysm was large at baseline, and the covered branch was probably hemodynamically functional over time, keeping the sac perfused. Both lesions showed some remodeling and shrinking during follow-up, but not enough to be considered a class 5 Cekirge-Saatci aneurysm. Further follow-up will be performed to look for further remodeling, stability, or regrowth.

More interestingly, we observed in 1 case an aneurysm recurrence after DSA- and MRI-proven occlusion, at 3.5 years after treatment (Fig. 3). To our knowledge, this is the first study reporting a recurrence after FD stent insertion for an MCA bifurcation aneurysm. The actual recurrence rate after FD placement on distal bifurcations may be underestimated. Most of the studies have a mean follow-up time of $<2$ years. Moreover, as happened in our population, as soon as occlusion is confirmed, DSA controls are commonly and (we thought) reasonably stopped. Nonetheless, we are not able to predict the evolution of 


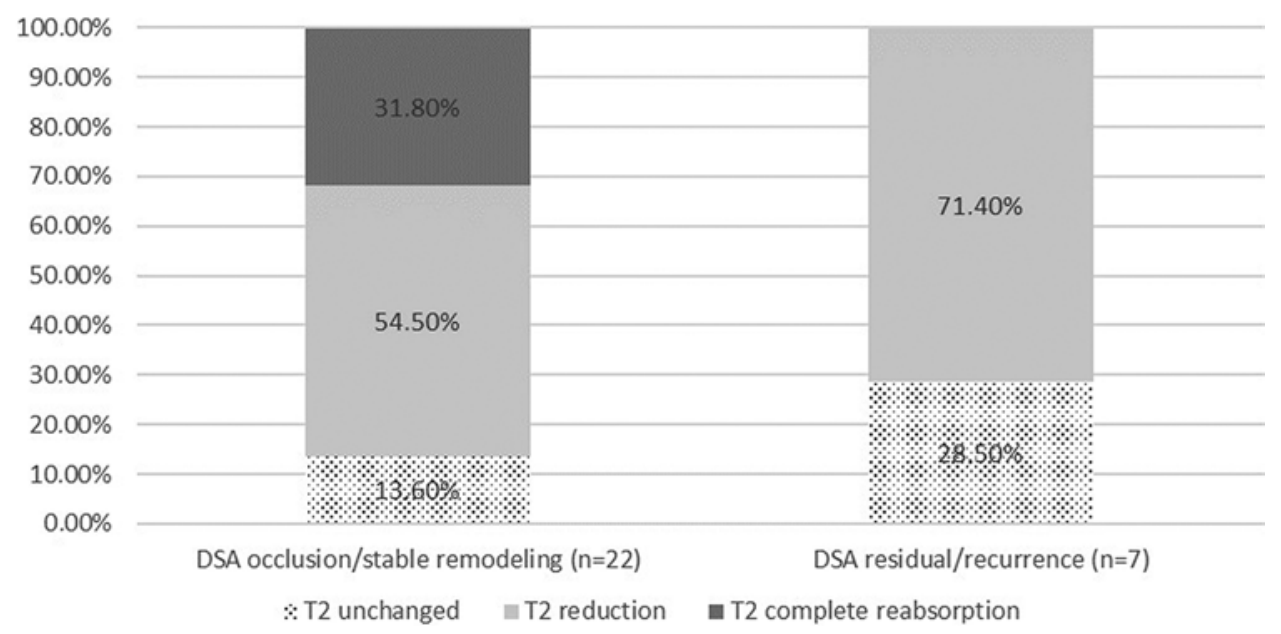

FIG. 5. DSA sac analysis results versus last MRI T2-weighted sequences; sac axial modifications at last follow-up. Aneurysms are subdivided into 2 groups according to last DSA results: first group $(n=22)$ including occlusion and stable remodeling, second group $(n=7)$ including residual lesion and recurrence. T2 sequence results are classified as complete reabsorption (dark gray), reduction of axial diameter (light gray), and unchanged (small dots); distribution is shown as a percentage within each group of patients.

this recurrence and the real hemorrhagic risk of such a recanalization. In any case, long-term MRI follow-up should be required, possibly on a 3-T machine with 3D TOF sequences. The small recanalization that we observed was well recognizable even on 3D TOF sequences (Fig. 3H). In our experience, only 3D TOF sequences obtained on 3-T machines show very high concordance with DSA results in examining residual flow in the aneurysm sac, even in the presence of an FD stent, although DSA remains the gold standard to evaluate intraluminal stent permeability. ${ }^{1}$

Concerning follow-up protocols, only a few articles have reported on a scheduled MRI control ${ }^{3,7,12,17,23}$ and actually, aneurysmal sac shrinkage is rarely analyzed. ${ }^{7}$ This is quite surprising, considering that many studies include large and giant aneurysms and the fact that it is well known that DSA does not show the modification of the size of the thrombosed part of the sac, besides some indirect signs, such as mass effect on adjacent branches, and coil cast modification. Our analysis has some limitations, because T2-weighted sequences are not volumetric and the main diameter and not the volume was considered. However, the evaluation was rarely difficult, and operators hardly ever disagreed. In case of uncertainty, mostly due to previous coiling, an aneurysm was considered unmodified. Only $37 \%$ of the aneurysms occluded on DSA were complete or reabsorbed on MRI. This could be explained by the fact that either the mechanism of reabsorption might be longer than our mean follow-up time or, on the contrary, lesions will never resorb completely. Longer follow-up and larger population studies are needed to correlate these data with the long-term hemorrhagic risk of a nonreabsorbed aneurysm.

Nevertheless, we had one recurrence in one of the few cases with DSA- and MRI-proven occlusion, so we can even question if occlusion on MRI is the definite proof of aneurysm cure after FD stent placement.

Our study has many limitations. The first of these is the inherent bias of a retrospective single-center design, reporting descriptive data on a relatively small and heterogeneous population. Second, this small population does not allow subgroup analysis by FD stent type.

\section{Conclusions}

Flow diversion of bifurcation aneurysms is feasible with low rates of permanent morbidity and mortality and high occlusion rates. Caliber reduction and asymptomatic occlusion of covered cortical branches as well as silent perforator strokes are common. Severe ischemic complications with no certain identifiable and predictable factors may occur, as well as recurrence. For these reasons, FD stent insertion should be considered a second-line treatment for bifurcation aneurysm, and MRI controls should be required in all patients to evaluate silent ischemic lesions and aneurysmal sac reabsorption or recurrence over time.

\section{References}

1. Attali J, Benaissa A, Soize S, Kadziolka K, Portefaix C, Pierot L: Follow-up of intracranial aneurysms treated by flow diverter: comparison of three-dimensional time-of-flight MR angiography (3D-TOF-MRA) and contrast-enhanced MR angiography (CE-MRA) sequences with digital subtraction angiography as the gold standard. J Neurointerv Surg 8:81-86, 2016

2. Cagnazzo F, Mantilla D, Lefevre PH, Dargazanli C, Gascou $\mathrm{G}$, Costalat V: Treatment of middle cerebral artery aneurysms with flow-diverter stents: a systematic review and meta-analysis. AJNR Am J Neuroradiol 38:2289-2294, 2017

3. Carneiro A, Rane N, Küker W, Cellerini M, Corkill R, Byrne $\mathrm{JV}$ : Volume changes of extremely large and giant intracranial aneurysms after treatment with flow diverter stents. Neuroradiology 56:51-58, 2014

4. Caroff J, Neki H, Mihalea C, D’Argento F, Abdel Khalek H, Ikka L, et al: Flow-diverter stents for the treatment of saccu- 
lar middle cerebral artery bifurcation aneurysms. AJNR Am J Neuroradiol 37:279-284, 2016

5. Cekirge HS, Saatci I: A new aneurysm occlusion classification after the impact of flow modification. AJNR Am J Neuroradiol 37:19-24, 2016

6. Chalouhi N, Starke RM, Yang S, Bovenzi CD, Tjoumakaris S, Hasan D, et al: Extending the indications of flow diversion to small, unruptured, saccular aneurysms of the anterior circulation. Stroke 45:54-58, 2014

7. Clarençon F, Di Maria F, Gabrieli J, Shotar E, Zeghal C, Nouet A, et al: Flow diverter stents for the treatment of anterior cerebral artery aneurysms: safety and effectiveness. Clin Neuroradiol 27:51-56, 2017

8. Colby GP, Bender MT, Lin LM, Beaty N, Huang J, Tamargo $\mathrm{R}$, et al: Endovascular flow diversion for treatment of anterior communicating artery region cerebral aneurysms: a singlecenter cohort of 50 cases. J Neurointerv Surg 9:679-685, 2017

9. Cottier JP, Pasco A, Gallas S, Gabrillargues J, Cognard C, Drouineau J, et al: Utility of balloon-assisted Guglielmi detachable coiling in the treatment of 49 cerebral aneurysms: a retrospective, multicenter study. AJNR Am J Neuroradiol 22:345-351, 2001

10. Dabus G, Grossberg JA, Cawley CM, Dion JE, Puri AS, Wakhloo AK, et al: Treatment of complex anterior cerebral artery aneurysms with Pipeline flow diversion: mid-term results. J Neurointerv Surg 9:147-151, 2017

11. Gascou G, Lobotesis K, Brunel H, Machi P, Riquelme C, Eker O, et al: Extra-aneurysmal flow modification following Pipeline Embolization Device implantation: focus on regional branches, perforators, and the parent vessel. AJNR Am J Neuroradiol 36:725-731, 2015

12. Gawlitza M, Januel AC, Tall P, Bonneville F, Cognard C: Flow diversion treatment of complex bifurcation aneurysms beyond the circle of Willis: a single-center series with special emphasis on covered cortical branches and perforating arteries. J Neurointerv Surg 8:481-487, 2016

13. Iosif C, Berg P, Ponsonnard S, Carles P, Saleme S, Ponomarjova $S$, et al: Role of terminal and anastomotic circulation in the patency of arteries jailed by flow-diverting stents: from hemodynamic changes to ostia surface modifications. J Neurosurg 126:1702-1713, 2017

14. Iosif C, Mounayer C, Yavuz K, Saleme S, Geyik S, Cekirge HS, et al: Middle cerebral artery bifurcation aneurysms treated by extrasaccular flow diverters: midterm angiographic evolution and clinical outcome. AJNR Am J Neuroradiol 38:310-316, 2017

15. Kallmes DF, Brinjikji W, Boccardi E, Ciceri E, Diaz O, Tawk R, et al: Aneurysm Study of Pipeline in an Observational Registry (ASPIRe). Intervent Neurol 5:89-99, 2016

16. Martínez-Galdámez M, Romance A, Vega P, Vega A, Caniego JL, Paul L, et al: Pipeline endovascular device for the treatment of intracranial aneurysms at the level of the circle of Willis and beyond: multicenter experience. J Neurointerv Surg 7:816-823, 2015

17. Morais R, Mine B, Bruyère PJ, Naeije G, Lubicz B: Endovascular treatment of intracranial aneurysms with the p64 flow diverter stent: mid-term results in 35 patients with 41 intracranial aneurysms. Neuroradiology 59:263-269, 2017

18. Nelson PK, Lylyk P, Szikora I, Wetzel SG, Wanke I, Fiorella D: The Pipeline Embolization Device for the intracranial treatment of aneurysms trial. AJNR Am J Neuroradiol 32:34-40, 2011
19. Nossek E, Zumofen DW, Setton A, Potts MB, Raz E, Shapiro $\mathrm{M}$, et al: Treatment of distal anterior cerebral artery aneurysms with the Pipeline Embolization Device. J Clin Neurosci 35:133-138, 2017

20. Pierot L, Moret J, Turjman F, Herbreteau D, Raoult H, Barreau $\mathrm{X}$, et al: WEB treatment of intracranial aneurysms: clinical and anatomic results in the French observatory. AJNR Am J Neuroradiol 37:655-659, 2016

21. Pistocchi S, Blanc R, Bartolini B, Piotin M: Flow diverters at and beyond the level of the circle of Willis for the treatment of intracranial aneurysms. Stroke 43:1032-1038, 2012

22. Rodríguez-Hernández A, Sughrue ME, Akhavan S, Habdank-Kolaczkowski J, Lawton MT: Current management of middle cerebral artery aneurysms: surgical results with a "clip first" policy. Neurosurgery 72:415-427, 2013

23. Saleme S, Iosif C, Ponomarjova S, Mendes G, Camilleri Y, Caire F, et al: Flow-diverting stents for intracranial bifurcation aneurysm treatment. Neurosurgery 75:623-631, 2014

24. Spiotta AM, Gupta R, Fiorella D, Gonugunta V, Lobo B, Rasmussen PA, et al: Mid-term results of endovascular coiling of wide-necked aneurysms using double stents in a Y configuration. Neurosurgery 69:421-429, 2011

25. Szikora I, Berentei Z, Kulcsar Z, Marosfoi M, Vajda ZS, Lee $\mathrm{W}$, et al: Treatment of intracranial aneurysms by functional reconstruction of the parent artery: the Budapest experience with the Pipeline Embolization Device. AJNR Am J Neuroradiol 31:1139-1147, 2010

26. Topcuoglu OM, Akgul E, Daglioglu E, Topcuoglu ED, Peker A, Akmangit I, et al: Flow diversion in middle cerebral artery aneurysms: is it really an all-purpose treatment? World Neurosurg 87:317-327, 2016

27. van Rooij WJ, Sluzewski M: Perforator infarction after placement of a Pipeline flow-diverting stent for an unruptured A1 aneurysm. AJNR Am J Neuroradiol 31:E43-E44, 2010

28. Vendrell JF, Menjot N, Costalat V, Hoa D, Moritz J, Brunel $\mathrm{H}$, et al: Endovascular treatment of 174 middle cerebral artery aneurysms: clinical outcome and radiologic results at long-term follow-up. Radiology 253:191-198, 2009

29. Yavuz K, Geyik S, Saatci I, Cekirge HS: Endovascular treatment of middle cerebral artery aneurysms with flow modification with the use of the Pipeline Embolization Device. AJNR Am J Neuroradiol 35:529-535, 2014

\section{Disclosures}

Dr. Cognard is a consultant for Medtronic, MicroVention, Stryker, and Balt.

\section{Author Contributions}

Conception and design: Michelozzi, Cognard. Acquisition of data: Michelozzi, Darcourt, Guenego, Januel, Cognard. Analysis and interpretation of data: Michelozzi, Darcourt, Cognard. Drafting the article: Michelozzi. Critically revising the article: Michelozzi, Darcourt. Reviewed submitted version of manuscript: all authors. Approved the final version of the manuscript on behalf of all authors: Michelozzi. Statistical analysis: Michelozzi. Study supervision: Cognard.

\section{Correspondence}

Caterina Michelozzi: Hôpital Pierre-Paul Riquet, Toulouse, France. caterina.michelozzi@gmail.com. 